\title{
Phase-covariant cloning of coherent states
}

\author{
Massimiliano F. Sacch? \\ QUIT Quantum Information Theory Group, CNISM and CNR - Istituto Nazionale per la Fisica della Materia, \\ Dipartimento di Fisica "A. Volta", University of Pavia, via A. Bassi 6, I-27100 Pavia, Italy, \\ Istituto Universitario di Studi Superiori (IUSS), via Luino 4, I-27100 Pavia, Italy.
}

(Dated: May 8, 2019)

\begin{abstract}
We consider the problem of phase-covariant cloning for coherent states. We show that an experimental scheme based on ideal phase measurement and feedforward outperforms the semiclassical procedure of ideal phase measurement and preparation in terms of fidelity. A realistic scheme where the ideal phase measurement is replaced with double-homodyne detection is shown to be unable to overcome the semiclassical cloning strategy. On the other hand, such a realistic scheme is better than semiclassical cloning based on double-homodyne phase measurement and preparation.
\end{abstract}

PACS numbers: 03.67.-a; 03.65.-w

\section{INTRODUCTION}

The impossibility of exact quantum cloning - i.e. copying the state of a quantum system to a larger number of uses [1] - has stimulated the search for quantum devices that can emulate cloning with the highest possible fidelity. Many optimal cloners have been found, since the simplest case of qubits $[2,3$, 4], including general finite-dimensional systems [5], restricted sets of input states [6, 7], and infinite-dimensional systems such as harmonic oscillators - the so called continuous variable cloners [8, 9]. More recently, cloning for mixed states has been considered, for both qubits [10] and Gaussian continuous variable systems [11].

In the present paper, we analyze the cloning of coherent states by phase-preserving maps. More precisely, this means that we will consider a set of coherent states with a fixed known absolute value of the amplitude and unknown phase, thus studying cloning machines that are covariant with respect to the phase Abelian group $U(1)$, instead of the Weyl-Heisenberg group of complex displacements, as done for arbitrary coherent states. Clearly, one expects to improve the fidelity of the $N$ to $M$ cloning of coherent states $F_{N M}=\frac{M N}{M N+M-N}[9]$, since a restricted set of states is considered.

The issue of quantum cloning is studied for both its fundamental interest and its relevance in practice, in the realm of quantum key distribution and quantum cryptography [12, 13]. In particular, the study of phase-covariant cloning may be of interest in analyzing the security of quantum cryptographic protocols based on phase coding. While extensive work has been devoted to phase-covariant cloning for qubits and qudits $[14,15,16,17,18,19,20,21]$, the case of coherent states is still unexplored.

The general theory for the problem of cloning that is covariant with respect to a given group of transformations has been given in $[\underline{6}]$. However, the optimal phase-

*URL: http://www.qubit.it covariant cloning for coherent states has never been derived. The main reason is that the phase-covariance property entails infinite equivalent representations, corresponding to Hilbert subspaces with fixed photon-number difference, which makes the problem highly untractable. The derivation of the optimal phase-covariant cloning would be interesting to give a benchmark for realizable cloning schemes, but presumably the result would be almost academic, beyond the experimental capability of the best quantum optics laboratories.

Here, we will study the problem of phase-covariant cloning of coherent states by considering experimental schemes based on splitting the input coherent states, phase measurement, and feedforward. Measurementand-feedforward schemes have been considered recently for cloning of coherent states and Gaussian mixed states [11, 22], where optimality has also been proved. We will compare such experimental schemes with the semiclassical cloning procedure based on phase estimation and preparation of coherent states.

The paper is organized as follows. In Sec. II we sketch the general problem for phase-covariant cloning. In Sec. III we evaluate the average fidelity of semiclassical cloning based on measurement and preparation, considering both ideal phase measurement and double-homodyne detection. In Sec. IV we consider two cloning schemes based on splitting, phase measurement and feedforward, when the ideal phase measurement and the phase measurement achieved by marginal of double-homodyne detection are adopted. Clearly, for a realistic implementation, the second scheme is more interesting, and in fact the effect of nonunit quantum efficiency of detectors is also taken into account. In these schemes two parameters can be set free: the ratio of the splitting and the feedforward gain, and one can suitably optimize over them to maximize the fidelity. In Sec. V, we compare the semiclassical strategy with the feedforward schemes. The following result is obtained: when an ideal phase measurement is performed, the feedforward scheme outperforms the semiclassical method, whereas when doublehomodyne detection is adopted - a much simpler and viable measurement - the feedforward scheme is in prac- 
tice unable to overcome the semiclassical cloning, even for very high quantum efficiency. However, the realistic scheme is better then the corresponding semiclassical cloning based on double-homodyne detection and preparation. In Sec. VI we draw conclusions with closing remarks.

\section{OPTIMAL PHASE-COVARIANT CLONING}

The problem of optimal phase-covariant cloning for continuous variable systems can be posed as follows. Let us denote by $a\left(a^{\dagger}\right)$ the annihilation (creation) operator of a single-mode radiation field, with $\left[a, a^{\dagger}\right]=1$. We are given a set of $N$ input copies $\left(e^{i a^{\dagger} a \phi} \rho_{0} e^{-i a^{\dagger} a \phi}\right)^{\otimes N}$, where $\rho_{0}$ is known, and the phase $\phi \in[0,2 \pi]$ is unknown, and we are interested in looking for a physical transformation (i.e. a completely positive map $\mathcal{E}$ ) which produces $M$ output copies with maximum fidelity with respect to the input, independently of the value of the unknown phase $\phi$. The fidelity of the output clones will then be the same for any input phase.

The input-output description in terms of completely positive maps is equivalent 23] to a scheme where an ancilla is added, a unitary transformation is performed, and the ancilla is discarded (i.e. traced out). The latter scheme is the traditional approach to the problem of optimal quantum cloning [2].

The optimal map can be searched among the covariant ones that satisfy

$$
\begin{aligned}
& \mathcal{E}\left[\left(e^{i a^{\dagger} a \phi} \rho_{0} e^{-i a^{\dagger} a \phi}\right)^{\otimes N}\right] \\
&=\quad\left(e^{i a^{\dagger} a \phi}\right)^{\otimes M} \mathcal{E}\left(\rho_{0}^{\otimes N}\right)\left(e^{-i a^{\dagger} a \phi}\right)^{\otimes M} .
\end{aligned}
$$

The covariance property leads to a striking simplification of the problem [6], since one can exploit the theory of irreducible representation of groups. However, in the phase-covariant problem for continuous variables, the optimal solution has never been reported, since infinite equivalent representations appear when considering the phase Abelian group $U(1)$. In fact, it is useful to consider the Choi-Jamiołkowski bijective correspondence of completely positive $(\mathrm{CP})$ maps $\mathcal{E}$ from the input Hilbert space $\mathcal{H}_{\text {in }}$ to the output Hilbert space $\mathcal{H}_{\text {out }}$ and positive operators $R_{\mathcal{E}}$ acting on $\mathcal{H}_{\text {in }} \otimes \mathcal{H}_{\text {out }}$, which is given by the following expressions

$$
\begin{aligned}
& R_{\mathcal{E}}=I \otimes \mathcal{E}(|\Omega\rangle\langle\Omega|), \\
& \mathcal{E}(\rho)=\operatorname{Tr}_{\text {in }}\left[\left(\rho^{\tau} \otimes I_{\text {out }}\right) R_{\mathcal{E}}\right],
\end{aligned}
$$

where $|\Omega\rangle=\sum_{n=0}^{\infty}\left|\psi_{n}\right\rangle\left|\psi_{n}\right\rangle$ is a maximally entangled vector of $\mathcal{H}_{\text {in }}^{\otimes 2}$, and $\tau$ denotes the transposition on the fixed basis $\left\{\left|\psi_{n}\right\rangle\right\}$. In terms of the operator $R_{\mathcal{E}}$ the covariance property (11) can be written as

$$
\left[R_{\mathcal{E}},\left(e^{-i a^{\dagger} a \phi}\right)^{\otimes N} \otimes\left(e^{i a^{\dagger} a \phi}\right)^{\otimes M}\right]=0, \quad \forall \phi .
$$

The trace-preserving condition of the map $\mathcal{E}$ is given in terms of the positive operator $R_{\mathcal{E}}$ by the constraint

$$
\operatorname{Tr}_{\text {out }}\left[R_{\mathcal{E}}\right]=I_{\text {in }},
$$

and the global fidelity of the cloning machine is given by

$$
F=\operatorname{Tr}\left[\left(\rho^{\tau \otimes N} \otimes \rho^{\otimes M}\right) R_{\mathcal{E}}\right] .
$$

The optimal cloning machine can be obtained by maximizing $F$ in Eq. (5) under the constraints (3) and (4). The solution of such a problem is presently unknown.

\section{SEMICLASSICAL PHASE-COVARIANT CLONING}

The semiclassical strategy for cloning quantum states consists in performing a quantum measurement on the given input copies, and creating suitable output copies according to the measurement outcome. Then, for phasecovariant cloning, one can perform the ideal phase measurement to infer the phase of the unknown input with best accuracy.

The quantum estimation of the phase is the essential problem of high sensitive interferometry, and has received much attention in quantum optics [24]. For a singlemode electromagnetic field, the measurement cannot be achieved exactly, even in principle, due to the lack of a unique self-adjoint operator [25].

The most general and concrete approach to the problem of the phase measurement is quantum estimation theory [26]. Quantum estimation theory provides a more general description of quantum statistics in terms of POVM's (positive operator-valued measures) and gives the theoretical definition of an optimized phase measurement. The most powerful method for deriving the optimal phase measurement was given by Holevo 27] in the covariant case. In this way the optimal POVM for phase estimation has been derived for a single-mode field. More generally, the problem of estimating the phase shift has been addressed in Ref. [28] for any degenerate shift operator with arbitrary discrete spectrum.

The optimal POVM for the phase measurement can be written in terms of projectors on Susskind-Glogower states [29] as follows

$$
d \mu(\phi)=\frac{d \phi}{2 \pi}\left|e^{i \phi}\right\rangle\left\langle e^{i \phi}\right|,
$$

where $\left|e^{i \phi}\right\rangle=\sum_{n=0}^{\infty} e^{i \phi n}|n\rangle$, and $\{|n\rangle\}$ denotes the Fock basis. Notice that the states $\left|e^{i \phi}\right\rangle$ are not normalizable, neither are they orthogonal; however, they provide a resolution of the identity, and thus guarantee the completeness of the POVM, namely,

$$
\int_{0}^{2 \pi} d \mu(\phi)=I
$$


For a system in state $\rho$, the POVM in Eq. (6) gives the ideal phase distribution $p(\phi)$ according to Born's rule

$$
p(\phi)=\operatorname{Tr}[d \mu(\phi) \rho]=\frac{d \phi}{2 \pi}\left\langle e^{i \phi}|\rho| e^{i \phi}\right\rangle .
$$

For a set of $N$ input coherent states $|\alpha\rangle^{\otimes N}$, the semiclassical strategy consists in concentrating by a multisplitter the $N$ coherent states into a single coherent state with amplitude $\sqrt{N} \alpha$, performing the ideal phase measurement, and creating $M$ coherent states $\left|e^{i \phi}\right| \alpha|\rangle$, where $\phi$ is the phase-measurement outcome. Hence, on average one obtains the $M$ output copies

$$
\begin{aligned}
& \mathcal{E}\left(|\alpha\rangle\left\langle\left.\alpha\right|^{\otimes N}\right)\right. \\
= & \int_{0}^{2 \pi} \frac{d \phi}{2 \pi}\left|e^{i \phi}\right| \alpha|\rangle\left\langle\left. e^{i \phi}|\alpha|\right|^{\otimes M}\left|\left\langle\sqrt{N} \alpha \mid e^{i \phi}\right\rangle\right|^{2} .\right.
\end{aligned}
$$

Using the relations

$$
|\alpha\rangle=e^{-\frac{|\alpha|^{2}}{2}} \sum_{n=0}^{\infty} \frac{\alpha^{n}}{\sqrt{n !}}|n\rangle, \quad|\langle\alpha \mid \beta\rangle|^{2}=e^{-|\beta-\alpha|^{2}}
$$

we can evaluate the fidelity of each of the $M$ copies with the initial input as follows

$$
\begin{aligned}
F_{c l}^{S G} & =\int_{0}^{2 \pi} \frac{d \phi}{2 \pi}\left|\left\langle\alpha\left|e^{i \phi}\right| \alpha \mid\right\rangle\right|^{2}\left|\left\langle\sqrt{N} \alpha \mid e^{i \phi}\right\rangle\right|^{2} \\
& =\int_{0}^{2 \pi} \frac{d \phi}{2 \pi} e^{-|\alpha|^{2}[2(1-\cos \phi)+N]} \\
& \times \sum_{n, m=0}^{\infty} \frac{(\sqrt{N}|\alpha|)^{n+m}}{\sqrt{n ! m !}} e^{i \phi(n-m)} \\
& =e^{-|\alpha|^{2}(2+N)} \sum_{n, m=0}^{\infty} \frac{(\sqrt{N}|\alpha|)^{n+m}}{\sqrt{n ! m !}} I_{n-m}\left(2|\alpha|^{2}\right),
\end{aligned}
$$

where we used the identity

$$
\int_{0}^{2 \pi} \frac{d \phi}{2 \pi} e^{z \cos \phi+i m \phi}=I_{m}(z)
$$

$I_{m}(z)$ denoting the $m$-th order modified Bessel function.

In a realistic semiclassical cloning strategy, the ideal phase measurement is beyond the available technology [30]. Thus, we consider the case where double-homodyne detection is used instead, in order to estimate the phase. The probability distribution for the phase measurement of coherent states by means of double-homodyne detection with quantum efficiency $\eta$ is given in the Appendix. Using this result, the fidelity is then given by

$$
\begin{aligned}
F_{c l}^{D H} & =e^{-|\alpha|^{2}(2+N)} \sum_{n, m=0}^{\infty} \frac{\Gamma\left(\frac{n+m}{2}+1\right)}{n ! m !} \\
& \times\left(\frac{\sqrt{N}|\alpha|}{\sqrt{\Delta_{\eta}^{2}+1}}\right)^{n+m} I_{n-m}\left(2|\alpha|^{2}\right) .
\end{aligned}
$$

where $\Delta_{\eta}^{2}=\frac{1-\eta}{\eta}$.

As for any measure-and-prepare scheme, notice that the fidelities (12) and (13) do not depend on the number of output copies $M$. The schemes are clearly covariant, i.e. the fidelity depends just on $|\alpha|$, and not on the phase $\arg \alpha$.

\section{PHASE-COVARIANT CLONING VIA PARTIAL MEASUREMENT AND FEEDFORWARD}

In this Section we consider the following covariant cloning strategy based on phase measurement and feedforward. First, the $N$ coherent states are concentrated by a multisplitter into a single coherent state with amplitude $\sqrt{N} \alpha$. Second, this state is split into two coherent states by a beam splitter with tunable transmissivity, thus giving two coherent states $|\sqrt{N} \alpha \cos \theta\rangle$ and $|\sqrt{N} \alpha \sin \theta\rangle$, where $\theta \in[0, \pi]$. Third, a phase measurement described by a POVM $d \mu(\phi)$ is performed on the second coherent state $|\sqrt{N} \alpha \sin \theta\rangle$, and the displacement $D\left(k|\alpha| e^{i \phi}\right)$ is the feedforward input to the first coherent state $|\sqrt{N} \alpha \cos \theta\rangle$, where $\phi$ is the measurement outcome and $k$ a free parameter. Finally, this coherent state is split into $M$ coherent states whose amplitude is then rescaled by $\sqrt{M}$. By averaging over the phase-measurement outcomes, such a cloning strategy produces the following $M$ copies:

$$
\begin{aligned}
& \mathcal{E}\left(|\alpha\rangle\left\langle\left.\alpha\right|^{\otimes N}\right)=\int_{0}^{2 \pi} \frac{d \phi}{2 \pi}\right. \\
\times & \left|\frac{\sqrt{N} \alpha \cos \theta+k|\alpha| e^{i \phi}}{\sqrt{M}}\right\rangle\left\langle\left.\frac{\sqrt{N} \alpha \cos \theta+k|\alpha| e^{i \phi}}{\sqrt{M}}\right|^{\otimes M}\right. \\
\times & \langle\sqrt{N} \alpha \sin \theta|d \mu(\phi)| \sqrt{N} \alpha \sin \theta\rangle .
\end{aligned}
$$

The fidelity of each of the $M$ copies with the initial input depends on the parameters $\theta$ and $k$, along with the POVM $d \mu(\phi)$, and can be written as follows

$$
\begin{aligned}
F_{f f} & =\int_{0}^{2 \pi} \frac{d \phi}{2 \pi}\left|\left\langle\alpha \mid\left(\sqrt{N} \alpha \cos \theta+k|\alpha| e^{i \phi}\right) / \sqrt{M}\right\rangle\right|^{2} \\
& \times\langle\sqrt{N} \alpha \sin \theta|d \mu(\phi)| \sqrt{N} \alpha \sin \theta\rangle .
\end{aligned}
$$

\section{A. Using ideal phase measurement}

With a similar derivation of Eq. (12), when the ideal phase measurement (6) is adopted the fidelity in Eq. (15) is given by

$$
\begin{aligned}
F_{f f}^{S G} & =e^{-\frac{|\alpha|^{2}}{M}\left[(\sqrt{M}-\sqrt{N} \cos \theta)^{2}+N M \sin ^{2} \theta+k^{2}\right]} \\
& \times \sum_{n, m=0}^{\infty} \frac{(\sqrt{N}|\alpha| \sin \theta)^{n+m}}{\sqrt{n ! m !}} \\
& \times I_{n-m}\left[\frac{2|\alpha|^{2}}{M}(\sqrt{M}-\sqrt{N} \cos \theta) k\right]
\end{aligned}
$$




\section{B. Using double-homodyne detection}

Using Eq. (19) of the Appendix for the phase measurement of coherent states by means of double-homodyne detection, one obtains the following expression for the fidelity of the cloning based on double-homodyne detection and feedforward:

$$
\begin{aligned}
F_{f f}^{D H} & =e^{-\frac{|\alpha|^{2}}{M}\left[(\sqrt{M}-\sqrt{N} \cos \theta)^{2}+\frac{N M \sin ^{2} \theta}{\Delta_{\eta}^{2}+1}+k^{2}\right]} \\
& \times \sum_{n, m=0}^{\infty} \frac{(\sqrt{N}|\alpha| \sin \theta)^{n+m}}{\sqrt{\Delta_{\eta}^{2}+1}} \frac{\Gamma\left(\frac{n+m}{2}+1\right)}{n ! m !} \\
& \times I_{n-m}\left[\frac{2|\alpha|^{2}}{M}(\sqrt{M}-\sqrt{N} \cos \theta) k\right] .
\end{aligned}
$$

\section{NUMERICAL RESULTS}

The expression of the fidelities for the feedforwardbased schemes can be optimized versus the parameters $\theta$ and $k$, namely the beam splitter transmissivity and the gain factor of the displacement. In this Section we present some numerical results of the optimized fidelities, and compare the quality of the cloning with the semiclassical one.

In Fig. 1 we report the fidelity for 1-to-2 phasecovariant cloning of coherent states versus the absolute amplitude $|\alpha|$ : the feedforward scheme with ideal phase measurement (solid line), and double-homodyne phase measurement with quantum efficiency $\eta=1$ (dashed line) and $\eta=0.8$ (dotted line); semiclassical cloning via ideal phase measurement and preparation (dot-dashed line).

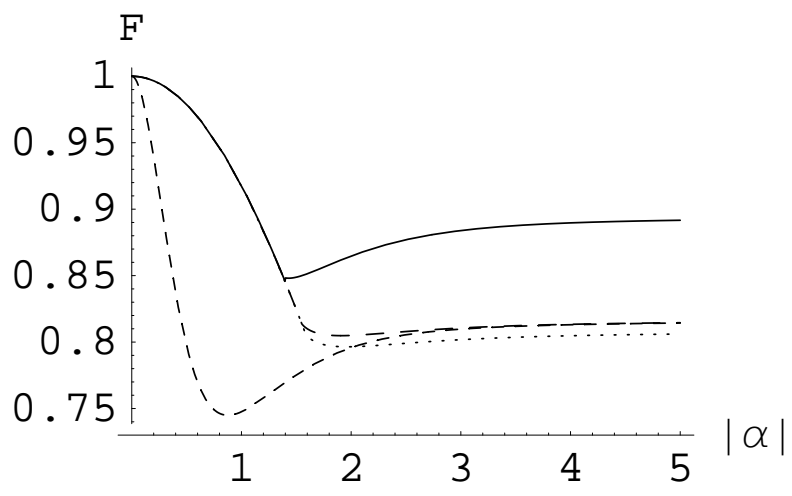

FIG. 1: Fidelity for 1-to-2 phase-covariant cloning of coherent states versus the absolute amplitude $|\alpha|$ : the feedforward scheme with ideal phase measurement (solid line), and doublehomodyne phase measurement with quantum efficiency $\eta=1$ (dashed line) and $\eta=0.8$ (dotted line); semiclassical cloning via ideal phase measurement and preparation (dot-dashed line).

It turns out that when the ideal phase measurement is performed, the feedforward scheme outperforms the semiclassical method, whereas when double-homodyne detection is adopted the feedforward scheme is in practice unable to do better than the semiclassical cloning, even for very high quantum efficiency. We notice that for low values of $|\alpha|$, i.e. $|\alpha| \lesssim 1.6$, the optimization of the feedforward schemes gives $\theta=k=0$, which means that cloning is achieved just by splitting the coherent states, where the phase is preserved. This can be easily understood since a coherent state with such a low intensity has a very poor phase resolution due to the high fidelity with the vacuum state, and hence a cloning strategy based on phase-measurement has a poor efficiency. On the other hand, for both the semiclassical and the feedforward cloning schemes, the fidelity rapidly saturates to a constant for $|\alpha| \geq 4$, independently of the amplitude. In all cases, as expected, the fidelity overcomes the value $2 / 3$ of the cloning for arbitrary coherent states. For $N$ to $M$ cloning, the above features are essentially unchanged.

In Fig. 2 we show the values of the fidelity for 1to-M phase-covariant cloning with $|\alpha|=5$ versus the output number of copies $M$, for the feedforward-scheme with both ideal phase measurement (gray) and doublehomodyne phase measurement with quantum efficiency $\eta=1$ (black). For increasing number of output copies, even the value of the fidelity of the feedforward scheme and ideal phase measurement tends to the classical value $F \simeq 0.8157$, confirming that quantum information becomes classical when distributed to many users [31].

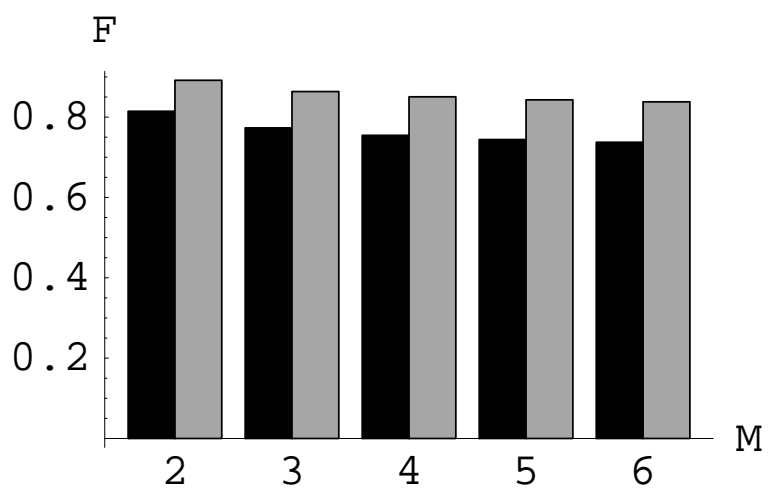

FIG. 2: Fidelity for 1-to-M phase-covariant cloning of coherent states with absolute amplitude $|\alpha|=5$ versus the output number of copies $M$ : feedforward-scheme with ideal phase measurement (gray), and double-homodyne phase measurement with quantum efficiency $\eta=1$ (black).

In Tab. 1, we report the explicit values for the fidelity of different 1-to-2 cloning schemes and for different values of $|\alpha|$, along with the optimal splitting parameter $\theta$ and gain parameter $k$ for the feedforward schemes. Although a realistic scheme based on double-homodyne detection and feedforward does not outperform the semiclassical cloning (i.e. $F_{f f}^{D H}<F_{c l}^{S G}$ ), however it does outperform semiclassical cloning based on double-homodyne detection and preparation (i.e. $F_{f f}^{D H}>F_{c l}^{D H}$ ). 


\section{CONCLUSIONS}

We have studied the problem of phase-covariant cloning of coherent states. After showing the difficulty of the problem of deriving the optimal phase-covariant cloning, we have compared cloning schemes based on phase measurement and feedforward with semiclassical schemes based on phase measurement and preparation. In both cases, we considered an ideal phase measurement and double-homodyne phase measurement. In the feedforward schemes we have shown that the fidelity can be optimized over the splitting parameter of the input copies and the feedforward gain. When the ideal phase measurement is performed, the feedforward scheme outperforms the semiclassical method, whereas when doublehomodyne detection is adopted the feedforward scheme is unable to overcome the semiclassical cloning, even for very high quantum efficiency. In any case, the realistic scheme is better then the corresponding semiclassical cloning based on double-homodyne detection and preparation.

\begin{tabular}{|c|ccc|ccc|c|c|}
\hline$|\alpha|$ & $F_{f f}^{S G}$ & $k$ & $\theta$ & $F_{f f}^{D H(\eta=0.8)}$ & $k$ & $\theta$ & $F_{c l}^{S G}$ & $F_{c l}^{D H}$ \\
\hline 3 & 0.884 & 0.746 & 0.861 & 0.802 & 0.697 & 0.809 & 0.810 & 0.703 \\
\hline 4 & 0.890 & 0.722 & 0.818 & 0.805 & 0.701 & 0.861 & 0.813 & 0.705 \\
\hline 5 & 0.892 & 0.714 & 0.802 & 0.806 & 0.703 & 0.793 & 0.814 & 0.706 \\
\hline 6 & 0.893 & 0.711 & 0.796 & 0.807 & 0.704 & 0.791 & 0.815 & 0.706 \\
\hline
\end{tabular}

TABLE I: Numerical values for the fidelity of 1-to-2 phasecovariant cloning of coherent states versus the absolute value of the amplitude $|\alpha|$ for different schemes: feedforward and ideal phase measurement $\left(F_{f f}^{S G}\right)$; feedforward and double-homodyne phase measurement with quantum efficiency $\eta=0.8\left(F_{f f}^{D H}\right)$; ideal phase measurement and preparation $\left(F_{c l}^{S G}\right)$; double-homodyne phase measurement and preparation $\left(F_{c l}^{D H}\right)$. For the feedforward schemes the optimized values of the gain parameter $k$ and the splitting parameter $\theta$ are also reported.

Our results show that an experimental verification of the quantum features of a phase-covariant cloning of coherent states is very demanding, since a feedforward scheme would require an ideal phase measurement, which per se is a hard task. Unfortunately, a phase measurement based on the customary double-homodyne detec- tion is too noisy to produce good clones of coherent states.

\section{Appendix}

The POVM describing double-homodyne detection (or, equivalently, heterodyne detection) with quantum efficiency $\eta$ is given by 32 ]

$$
d \mu(\alpha)=\frac{d^{2} \alpha}{\pi} \int_{\mathbb{C}} \frac{d^{2} \beta}{\pi \Delta_{\eta}^{2}} e^{-\frac{|\beta-\alpha|^{2}}{\Delta_{\eta}^{2}}}|\beta\rangle\langle\beta|,
$$

where $\Delta_{\eta}^{2}=\frac{1-\eta}{\eta}$, and $d^{2} \alpha=d \operatorname{Re} \alpha d \operatorname{Im} \alpha=|\alpha| d|\alpha| d \phi$ with $\alpha=|\alpha| e^{i \phi}$. The probability distribution $p(\phi \mid \gamma)$ for the phase measurement on a coherent state $|\gamma\rangle$ is then obtained as a marginal integration over the modulus $|\alpha|$ of the measurement outcome $\alpha$, and one has

$$
\begin{aligned}
p(\phi \mid \gamma) & =\frac{d \phi}{\pi} \int_{0}^{\infty} d|\alpha||\alpha| \int_{\mathbb{C}} \frac{d^{2} \beta}{\pi \Delta_{\eta}^{2}} e^{-\frac{|\beta-\alpha|^{2}}{\Delta_{\eta}^{2}}}|\langle\gamma \mid \beta\rangle|^{2} \\
& =\frac{d \phi}{\pi} \int_{0}^{\infty} d|\alpha||\alpha| \int_{\mathbb{C}} \frac{d^{2} \beta}{\pi \Delta_{\eta}^{2}} e^{-\frac{|\beta-\gamma|^{2}}{\Delta_{\eta}^{2}}} e^{-|\beta-\alpha|^{2}} \\
& =\frac{d \phi}{\pi\left(\Delta_{\eta}^{2}+1\right)} \int_{0}^{\infty} d|\alpha||\alpha| e^{-\frac{|\alpha-\gamma|^{2}}{\Delta_{\eta}^{2}+1}} \\
& =\frac{d \phi}{2 \pi} e^{-\frac{|\gamma|^{2}}{\Delta_{\eta}^{2}+1}} \int_{0}^{\infty} d t e^{-t} e^{\sqrt{\frac{t}{\Delta_{\eta}^{2}+1}}\left(e^{i \phi} \gamma^{*}+e^{-i \phi} \gamma\right)} \\
& =\frac{1}{2 \pi} e^{-\frac{|\gamma|^{2}}{\Delta_{\eta}^{2}+1}} \sum_{n, m=0}^{\infty} \frac{\Gamma\left(\frac{n+m}{2}+1\right)}{n ! m !} \\
& \times \frac{\gamma^{* n} \gamma^{m}}{\left(\Delta_{\eta}^{2}+1\right)^{\frac{n+m}{2}}} e^{i \phi(n-m)}
\end{aligned}
$$

where $\Gamma(x)=\int_{0}^{\infty} d t e^{-t} t^{x-1}$ denotes the usual gamma function.

\section{Acknowledgments}

This work has been sponsored by Ministero Italiano dell'Università e della Ricerca (MIUR) through FIRB (2001) and PRIN 2005.
[1] W. K. Wootters and W. H. Zurek, Nature 299, 802 (1982); D. Dieks, Phys. Lett. A, 92, 271 (1982); H. P. Yuen, Phys. Lett. A 113, 405 (1986); G. C. Ghirardi and T. Weber, Nuovo Cimento B 78, 9 (1983).

[2] V. Bužek and M. Hillery, Phys. Rev. A 54, 1844 (1996).

[3] N. Gisin and S. Massar, Phys. Rev. Lett. 79, 2153 (1997).

[4] D. Bruss, D. P. DiVincenzo, A. Ekert, C. A. Fuchs, C. Macchiavello, and J. A. Smolin, Phys. Rev. A 57, 2368 (1998).
[5] R. F. Werner, Phys. Rev. A 58, 1827 (1998); R. F. Werner and M. Keyl, J. Math. Phys. 40, 3283 (1999).

[6] G. M. D'Ariano and P. Lo Presti, Phys. Rev. A 64, 042308 (2001).

[7] G. M. D'Ariano and C. Macchiavello, Phys. Rev. A 67, 042306 (2003).

[8] N. J. Cerf, A. Ipe, and X. Rottenberg, Phys. Rev. Lett. 85, 1754 (2000); N. J. Cerf and S. Iblisdir, Phys. Rev. Lett. 87, 247903 (2001). 
[9] S. L. Braunstein, N. J. Cerf, S. Iblisdir, P. van Loock, and S. Massar, Phys. Rev. Lett. 86, 4938 (2001).

[10] G. M. D'Ariano, C. Macchiavello, and P. Perinotti, Phys. Rev. Lett. 95, 060503 (2005).

[11] G. M. D'Ariano, P. Perinotti, and M. F. Sacchi, New J. Phys. 8, 99 (2006); Europhys. Lett. 75, 195 (2006); quant-ph/0609046

[12] C. H. Bennett and G. Brassard, in Proceedings of the IEEE International Conference on Computers, Systems, and Signal Processing, Bangalore, India (IEEE, New York, 1984), p. 175; C. H. Bennett, Phys. Rev. Lett. 68, 3121 (1992); N. Gisin, G. Ribordy, W. Tittel, and H. Zbinden, Rev. Mod. Phys. 74, 145 (2002).

[13] A. K. Ekert, Phys. Rev. Lett. 67, 661 (1991); Nature 358, 14 (1992).

[14] D. Bruss, M. Cinchetti, G. M. D'Ariano, and C. Macchiavello, Phys. Rev. A 62, 012302 (2000).

[15] V. Karimipour and A. T. Rezakhani, Phys. Rev. A 66, 052111 (2002).

[16] H. Fan, K. Matsumoto, X. B. Wang, and M. Wadati, Phys. Rev. A 65, 012304 (2001).

[17] H. Fan, H. Imai, K. Matsumoto, and X. B. Wang, Phys. Rev. A 67, 022317 (2003).

[18] J. Fiurášek, Phys. Rev. A 67, 052314 (2003).

[19] F. Buscemi, G. M. D'Ariano, and C. Macchiavello, Phys. Rev. A 71, 042327 (2005).

[20] F. Sciarrino and F. De Martini, Phys. Rev. A 72, 062313
(2005).

[21] F. Buscemi, G. M. D'Ariano, C. Macchiavello, and P. Perinotti, Phys. Rev. A 74, 042309 (2006).

[22] U. L. Andersen, V. Josse, and and G. Leuchs, Phys. Rev. Lett. 94, 240503 (2005).

[23] F. Buscemi, G. M. D'Ariano, and M. F. Sacchi, Phys. Rev. A 68, 042113 (2003).

[24] Physica Scripta T 48 (1993) (special issue on Quantum Phase and Phase Dependent measurements).

[25] F. London, Z. Phys. 37, 915 (1926); 40, 193 (1927).

[26] C. W. Helstrom, Quantum Detection and Estimation Theory (Academic, New York, 1976).

[27] A. S. Holevo. Probabilistic and statistical aspects of quantum theory (North-Holland, Amsterdam, 1982).

[28] G. M. D'Ariano, C. Macchiavello, and M. F. Sacchi, Phys. Lett. A 248, 103 (1998).

[29] L. Susskind and J. Glogower, Physics 1, 49 (1964).

[30] A large class of theoretical schemes, however, have been proposed in F. Buscemi, G. M. D'Ariano, and M. F. Sacchi, Phys. Lett. A 312, 315 (2003).

[31] G. Chiribella and G. M. D'Ariano, Phys. Rev. Lett. 97, 250503 (2006).

[32] See, for example, G. M. D'Ariano, M. G. A. Paris, and M. F. Sacchi, Quantum Tomography, in Advances in Imaging and Electron Physics 128, 205-308 (2003), quant-ph/0302028. 